\title{
Biomarcadores de fibrosis y función ventricular derecha en maratonistas con distinto grado de entrenamiento: estudio en la Maratón de Santiago.
}

\author{
J. Rodrigo Fernández ${ }^{1}$, María Paz Ocaranza ${ }^{1,2}$, Sebastián Herrera ${ }^{1}$, \\ Julián Vega ${ }^{1}$, Manuel Salinas ${ }^{1}$, Felipe Contreras-Briceño ${ }^{3}$, \\ Silvana Llevaneras ${ }^{1}$, Jorge E. Jalil ${ }^{1}$, Fernando Yañez ${ }^{1}$, Paz Godoy ${ }^{1}$, \\ Samuel Córdova ${ }^{1}$, Mario Chiong ${ }^{4,5}$, Mario Muñoz $^{6}$, Sergio Lavandero ${ }^{4,5,7}$, \\ Pablo Castro ${ }^{1,2}$, Luigi Gabrielli. ${ }^{1,2}$
}

\begin{abstract}
1 División de Enfermedades Cardiovasculares, Facultad Medicina, Pontificia Universidad Católica de Chile. 2 Advanced Center for Chronic Diseases (ACCDiS). Facultad de Medicina, Pontificia Universidad Católica de Chile. 3 Kinesiología. Departamento Ciencias de la Salud, Pontificia Universidad Católica de Chile.

4 Departamento de Bioquímica y Biología Molecular, Facultad Ciencias Químicas y Farmacéuticas, Pontificia Universidad Católica de Chile. 5 Advanced Center for Chronic Diseases (ACCDiS). Facultad de Ciencias Químicas y Farmacéuticas y Facultad de Medicina, Universidad de Chile, Santiago, Chile.

6 Hospital del Trabajador. Asociación Chilena de Seguridad, Santiago, Chile

7 Cardiology Division, Department of Internal Medicine. University of Texas Southwestern Medical Center, Dallas, Texas, USA.
\end{abstract}

Introducción: Atletas altamente entrenados muestran cambios cardíacos estructurales como adaptación a la sobrecarga, producto del ejercicio repetitivo y extenuante. Se han evidenciado elevación de biomarcadores de remodelado y fibrosis miocárdica posterior al ejercicio intenso en atletas. Sin embargo, el comportamiento de estos biomarcadores según el nivel de entrenamiento previo no se ha evaluado.

Objetivo: Investigar biomarcadores de fibrosis y función ventricular derecha en maratonistas con distinto nivel de entrenamiento previo.

Métodos: Se incluyeron 36 maratonistas hombres, sanos, que completaron $42 \mathrm{~km}$ en la maratón de Santiago. Se dividieron según entrenamiento previo en dos grupos, Grupo 1 (G1): $\geq 100$ km/semana y Grupo 2 (G2): $<100 \mathrm{~km} / \mathrm{semana}$. Se realizó ecocardiografía transtorácica y se evaluaron niveles plasmáticos de galectina-3 y del propéptido amino terminal del procolágeno tipo III (PIIINP) en la semana previa a la carrera e inmediatamente posterior a ésta.

Resultados: Posterior a la maratón, la función sistólica del ventrículo derecho disminuyó en el grupo G2 junto con un aumento significativo de los niveles plasmáticos de PIIIPNP $(61 \pm 16$ a $94 \pm 24 \mathrm{ng} / \mathrm{mL}, \mathrm{p}=0,01)$. Estos cambios no se observaron en el grupo G1 (65 \pm 11 a $90 \pm 29 \mathrm{ng} / \mathrm{mL}, \mathrm{p}=0,10)$. Los niveles plasmáticos de galectina-3 aumentaron significativamente en ambos grupos posterior al ejercicio $(6,8 \pm 2,2$ a $19,7 \pm 4,9$ $\mathrm{ng} / \mathrm{mL}, \mathrm{p} 0,012$ y $6,0 \pm 1,1$ a $19,4 \pm 5,9 \mathrm{ng} / \mathrm{mL}, \mathrm{p} 0,01$ ) en los grupos G1 y G2, respectivamente).

Conclusiones: Atletas con menor grado de entrenamiento, presentan posterior a una maratón un significativo aumento de productos de degradación del colágeno (PIIIPNP) asociado a disminución de la función del ventrículo derecho. Los niveles de galectina-3 plasmática aumentan significativamente en ambos grupos post-esfuerzo independiente del entrenamiento previo.

\section{Correspondencia:}

Dr. Luigi Gabrielli.

Igabrielli@med.puc.cl 


\section{Fibrosis and right ventricular function biomarkers in marathon runners: a study at the Santiago, Chile marathon.}

Introduction: Highly trained athletes show structural cardiac changes as adaptation to overload. Rise in remodeling biomarkers and myocardial fibrosis after intense exercise in athletes has been evidenced; however, the behavior of these biomarkers according to pre-competition training level has not been evaluated.

Objective: To evaluate fibrosis biomarkers levels and right ventricle function in marathon runners according to their previous training level, in the period prior to a marathon race and immediately after it.

Methods: Thirty-six healthy male marathon runners were included. Subjects were grouped according to their previous training level: Group $1(\mathrm{G} 1): \geq 100$ $\mathrm{km} /$ week and Group 2 (G2): $<100 \mathrm{~km} /$ week. Transthoracic echocardiography along with plasmatic levels of galectin-3 and amino terminal propeptide of type III procollagen (PIIINP) were measured one week previous and immediately after the marathon.

Results: Post-effort right ventricle systolic function decreased in $\mathrm{G} 2$, together with a significant elevation of PIIIPNP ( $61 \pm 16$ to $94 \pm 24 \mathrm{ng} / \mathrm{mL}, \mathrm{p}=0.01)$. These changes were not observed in G1 (from $65 \pm 11$ to $90 \pm 29 \mathrm{ng}$ / $\mathrm{mL}, \mathrm{p}=0.10$ ). Plasma galectin- 3 increased significantly in both groups immediately post-exercise $(6.8 \pm 2.2$ to $19.7 \pm 4.9 \mathrm{ng} / \mathrm{mL}, \mathrm{p}=0.012$, and $6.0 \pm 1.1$ to $19.4 \pm 5.9 \mathrm{ng} /$ $\mathrm{mL}, \mathrm{p}=0.01$, in $\mathrm{G} 1$ and $\mathrm{G} 2$. respectively).

Conclusion: Less trained athletes evidenced higher post marathon levels of PIIIPNP which is associated with a decreased global right ventricle function. Plasma galectin-3 levels increased significantly after intense exertion regardless of the intensity of previous training.

Keywords: excersice; biomarkers; fibrosis; right ventricle; myocardial injury 


\section{Introducción:}

Los atletas altamente entrenados experimentan cambios cardíacos estructurales y funcionales como un mecanismo de adaptación a una condición de sobrecarga, producto del ejercicio repetitivo y extenuante a lo largo del tiempo ${ }^{1}$, condición denominada "corazón de atleta". Estos cambios consisten en el incremento del tamaño y grosor de ambos ventrículos, lo que puede estar asociado a hipertrabeculación, simulando incluso una no compactación $^{2-4}$. Este aumento en los volúmenes de las cavidades cardíacas permite una mejor adaptación y aumento del gasto cardíaco durante el ejercicio ${ }^{5}$.

Una mayor repercusión sobre el ventrículo derecho respecto al izquierdo se ha observado en los deportes de resistencia, observándose una disfunción transitoria inmediatamente después del ejercicio, probablemente, por ser una cámara menos preparada para sobrecargas de presión y volumen ${ }^{6}$. Este efecto podría deberse a que las paredes del ventrículo derecho (VD) son más delgadas, siendo más susceptibles al remodelado. Asimismo, estos cambios pueden ir acompañados de fibrosis del tejido miocárdico $^{7}$ y una mayor incidencia de arritmias cardíacas ${ }^{8}$. La fibrosis miocárdica se define por un aumento significativo en el volumen de colágeno del tejido miocárdico, producto de lesión tisular secundaria a isquemia, inflamación y sobrecarga del miocardio ${ }^{9}$. La fibrosis miocárdica produce un aumento de la rigidez miocárdica ${ }^{10}$, incrementando la presión de fin de diástole. Además, ella se asocia con una reducción de la función sistólica, arritmias y eventos clínicos adversos ${ }^{11}$. Ante similares niveles de sobrecarga, la fibrosis miocárdica se ha pesquisado en algunos atletas de alto rendimiento, generando interrogantes sobre su relación con el ejercicio de resistencia y sus eventuales implicancias.

En base a estos antecedentes se ha estudiado la utilidad potencial de biomarcadores que traducen fibrosis miocárdica, así como la carga fibrótica acumulativa en atletas de resistencia. La galectina-3, desempeña un importante rol en el desarrollo de la fibrosis cardíaca al activar fibrocitos y macrófagos miocárdicos ${ }^{12}$. Además, galectina-3 actúa como un regulador de la inflamación aguda y crónica y se encuentra tanto en fibroblastos del miocardio como también en otras células y tejidos ${ }^{13}$. Estudios recientes en atletas de resistencia han mostrado que galectina- 3 aumenta significativamente tras un ejercicio intenso ${ }^{14}$. El propéptido amino-terminal del procolágeno tipo III (PIIINP) es un marcador de la síntesis, depósito y degradación del colágeno tipo IIII ${ }^{15}$. Por lo tanto, el aumento de sus concentraciones en suero refleja el aumento de estos procesos, lo que se ha evidenciado en afecciones inflamatorias, procesos fibróticos y remodelado ventricular ${ }^{16}$. En los últimos años, se ha estudiado la elevación de biomarcadores de remodelado y fibrosis miocárdica en respuesta aguda posterior al ejercicio intenso de ciertos atletas ${ }^{17}$. Sin embargo, el comportamiento de estos biomarcadores según el nivel de entrenamiento (adaptación y daño acumulado) previo a una maratón no ha sido evaluado. El objetivo del presente trabajo consistió en evaluar biomarcadores de fibrosis (galectina-3 y PIIINP) y función ventricular izquierda y derecha en corredores de la maratón de Santiago, con distinta intensidad de entrenamiento previo a la carrera e inmediatamente posterior a la misma.

\section{Métodos:}

Diseño del estudio. Estudio prospectivo (longitudinal con dos puntos de evaluación), simple ciego. Se estudiaron 36 corredores de maratón activos, de sexo masculino, edad entre 18 y 50 años. Para la inclusión, se consideró necesarias 3 a 5 participaciones en una maratón completa en los últimos 5 años. De acuerdo con la intensidad de entrenamiento, se dividieron en dos grupos:

a) Grupo I: Alto entrenamiento, carga de entrenamiento igual o mayor de $100 \mathrm{~km} / \mathrm{semana}$. (n=16).

b) Grupo II: Moderado entrenamiento, carga de entrenamiento máxima de 50 a $99 \mathrm{~km} / \mathrm{semana}$. $(\mathrm{n}=20)$.

Este estudio se adhirió a los principios de la Declaración de Helsinki y fue aprobado por el Comité de Ética para la Investigación Humana en la Facultad de Medicina de la Pontificia Universidad Católica de Chile. Se obtuvo un consentimiento informado por escrito de todos los participantes antes de cualquier procedimiento.

Los criterios de exclusión fueron: hipertensión arterial (presión arterial en reposo >140/90 mmHg en 2 instancias separadas), dislipidemia (colesterol total $>200 \mathrm{mg} /$ $\mathrm{dL}, \mathrm{LDL}>100 \mathrm{mg} / \mathrm{dL}, \mathrm{HDL}<40 \mathrm{mg} / \mathrm{dL}$, triglicéridos $>150 \mathrm{mg} / \mathrm{dL}$ ), diabetes mellitus, resistencia a la insulina (HOMA >2.5), tabaquismo en cualquier grado, enfermedad cerebrovascular previa (antecedente clínico auto reportado), consumo diario de alcohol $>40 \mathrm{~g} / \mathrm{día}$, uso de drogas, deterioro de la función renal (tasa de filtración glomerular $<60 \mathrm{~mL} / \mathrm{min}$ MDRD), historia familiar de muerte súbita, daño hepático, enfermedad autoinmune, neoplasia activa, enfermedad pulmonar obstructiva crónica, enfermedades que alteren niveles de los biomar- 
cadores estudiados (inflamación aguda o enfermedad infecciosa en el mes previo a la maratón) y el uso de los siguientes medicamentos: cualquier antihipertensivo, anorexígenos, antidepresivos y/o antibióticos.

Evaluación ecocardiográfica. En todos los sujetos se realizó una ecocardiografía transtorácica según las guías de la Sociedad Americana de Ecocardiografía (SAE) durante el período de entrenamiento e inmediatamente posterior a la maratón. El examen lo realizó un operador entrenado, incluyendo vistas paraesternales y apicales mediante un transductor de matriz de fase de 2,5 a 5 $\mathrm{MHz}$ (VIVID 7; GE Medical Systems, Horten, Noruega). Se evaluaron los grosores parietales, los volúmenes cavitarios y la masa ventricular izquierda de acuerdo con las recomendaciones de la $\mathrm{SAE}^{18}$. La función sistólica de ambos ventrículos se calculó utilizando para el ventrículo izquierdo el método de Simpson biplano, expresándose como fracción de eyección (FEVI); mientras que para el ventrículo derecho se calculó el cambio de área fraccional (CAF); y el strain de la pared libre y global del ventrículo derecho mediante strain bidimensional ("spleckle tracking") ${ }^{19}$; el desplazamiento del anillo tricuspídeo (TAPSE) mediante modo M y la velocidad sistólica del anillo lateral tricuspídeo mediante Doppler tisular. Las imágenes se adquirieron a una velocidad de cuadro de al menos $60 \mathrm{~Hz}$.

Evaluación de biomarcadores. En todos los sujetos se tomó una muestra sérica mediante punción directa de una vena ante cubital por una enfermera capacitada. Las muestras se colectaron previo a la carrera e inmediatamente posterior a ésta, en la línea de meta. Los niveles séricos de los biomarcadores PIIINP y galectina 3 se determinaron mediante un ensayo inmunoenzimático comercial (ELISA). Cada muestra se analizó por duplicado y el valor medio se obtuvo utilizando el método de curva estándar. Para la galectina-3 se usó el kit ELISA (R\&D system). Los límites de detección fueron $16 \mathrm{ng} /$ $\mathrm{ml}$ con un coeficiente de variación intraensayo de 3,8\% e interensayo de 6,3\%. Para PIIINP se usó el ensayo inmunoenzimático comercial de Cisbio Bioassays. Cada muestra se analizó por duplicado y se cuantificó por curva estándar, el límite de detección de PIIINP fue 36 ng/L con un coeficiente de variación intraensayo de $2,7 \%$ e interensayo de 7,3\%. Los recuentos de células sanguíneas, función hepática, renal y electrolitos plasmáticos se midieron por métodos estándar.
Análisis estadístico. La distribución normal se evaluó con la prueba de Shapiro-Wilk. Las variables dependientes se evaluaron mediante la prueba de Wilcoxon y las independientes con Mann-Whitney U test. La asociación entre variables se evaluó con el método de Spearman. Se consideró significativo un valor $\mathrm{p}<0,05$.

\section{Resultados:}

Se incluyeron 16 y 20 individuos en los grupos de mayor y menor entrenamiento, respectivamente. Las características clínicas de los atletas se desglosan en la Tabla 1. Ambos grupos tenían similar edad y no se observaron diferencias en parámetros sanguíneos y bioquímicos generales. Los biomarcadores PIIINP y galectina-3 basales no mostraron diferencias entre los grupos. Todos los sujetos finalizaron la carrera sin presentar eventos ni síntomas adversos.

\begin{tabular}{llll}
\multicolumn{3}{l}{ Tabla 1. Características generales basales de los sujetos } & \\
& $<100 \mathrm{~km} / \mathrm{sem}$ & $\geq 100 \mathrm{~km} / \mathrm{sem}$ & $\mathrm{p}$ \\
$\mathrm{N}$ & 20 & 16 & \\
Edad (años) & $39 \pm 5$ & $37 \pm 6$ & $\mathrm{NS}$ \\
Masculino (\%) & 100 & 100 & $\mathrm{NS}$ \\
Altura (cm) & $174 \pm 6$ & $172 \pm 7$ & $\mathrm{NS}$ \\
Peso (kg) & $73 \pm 8$ & $69 \pm 8$ & $\mathrm{NS}$ \\
Hematocrito (\%) & $43 \pm 3$ & $43 \pm 2$ & $\mathrm{NS}$ \\
ASC (m2) & $1,88 \pm 0.12$ & $1,82 \pm 0,13$ & $\mathrm{NS}$ \\
Sodio mEq/L & $142 \pm 2$ & $142 \pm 3$ & $\mathrm{NS}$ \\
Creatinina (mg/dL) & $0,97 \pm 0.10$ & $0,98 \pm 0,09$ & $\mathrm{NS}$ \\
AST U/L & $28 \pm 8$ & $29 \pm 9$ & $\mathrm{NS}$ \\
Ácido úrico mg/dL) & $5,0 \pm 0,9$ & $5,6 \pm 0,9$ & $\mathrm{NS}$ \\
PIIINP (ng/mL) & $61 \pm 16$ & $65 \pm 11$ & $\mathrm{NS}$ \\
Galectina-3 (ng/mL) & $6,0 \pm 1,1$ & $6,8 \pm 2,2$ & $\mathrm{NS}$ \\
&
\end{tabular}

ASC: Área de superficie corporal Hb: hemoglobina AST: aspartato aminotransferasa PIIINP: péptido amino terminal procolágeno III.

Ejercicio y remodelado cardíaco. En el grupo de sujetos de mayor entrenamiento se observaron cambios estructurales significativos con respecto al grupo de sujetos menos entrenados. En comparación con el Grupo 2, los individuos del G1 presentaron mayores diámetros diastólicos $(\mathrm{p}=0,04$, grosor de las paredes ventriculares, septal $(\mathrm{p}=0,005, y$ posterior $(\mathrm{p}=0,012$, masa ventricular izquier$\mathrm{da}(\mathrm{p}=0,001$, y volumen auricular izquierdo $(\mathrm{p}=0,001)$, (Tabla 2).

Efectos del ejercicio en la función ventricular. La FEVI se elevó significativamente y de forma similar en 


\begin{tabular}{|l|l|l|l|}
\hline \multicolumn{3}{|c|}{ Tabla 2. Características ecocardiográficas basales } \\
\hline & $<100 \mathrm{~km} / \mathrm{sem}$ & $\geq 100 \mathrm{~km} / \mathrm{sem}$ & P \\
\hline DTD VI (mm) & $50 \pm 5$ & $58 \pm 4^{*}$ & $0,04^{*}$ \\
\hline DTS VI (mm) & $30 \pm 4$ & $33 \pm 5$ & 0,4 \\
\hline PS VI (mm) & $9,0 \pm 1,6$ & $10,2 \pm 1,2^{*}$ & $0,005^{*}$ \\
\hline PP VI (mm) & $8,5 \pm 1,2$ & $9,3 \pm 2,1^{*}$ & $0,012^{*}$ \\
\hline FEVI (\%) & $55 \pm 6$ & $54 \pm 3$ & 0,11 \\
\hline IMVI (g/m2) & $78 \pm 18$ & $106 \pm 27^{*}$ & $0,001^{*}$ \\
\hline VAI (mL/m2) & $30 \pm 11$ & $42 \pm 8 *$ & $0,001^{*}$ \\
\hline
\end{tabular}

DTD: diámetro tele diastólico. DTS: diámetro tele sistólico. PS: pared septal. PP: pared posterior. VI: ventrículo izquierdo. FEVI: fracción de eyección del VI. FAC: cambio de área fraccional del VD: IMVI: índice de masa VI. VAI: volumen auricular izquierdo. * $p<0.05$ vs grupo $<100 \mathrm{~km} / \mathrm{sem}$.

los individuos de ambos grupos ( $\mathrm{p}=0,0001$, Tabla 3 ). Sin embargo, en los sujetos del G2 se observó caída de la función sistólica del ventrículo derecho, tanto por cambio de área fraccional $(55 \pm 10$ a $42 \% \pm 18$, p 0,03$)$ como por deformación de la pared libre del VD $(-24,0 \pm 3,6$ a $-19 \% \pm 4,8, \mathrm{p}$ 0,007). La deformación global del VD, TAPSE y la velocidad sistólica del anillo tricuspídeo lateral no presentaron diferencias significativas (Tabla 3).

\section{Efectos del ejercicio en biomarcadores de fibrosis} miocárdica. Los niveles plasmáticos basales de galectina-3 no mostraron diferencias entre los atletas del G1 y $\mathrm{G} 2(\mathrm{p}=0,369)$. En ambos grupos se observó un aumento significativo de la galectina-3 post-maratón sin diferencias entre G1 y G2 $(6,8 \pm 2,2$ a $19,7 \pm 4,9 \mathrm{ng} / \mathrm{mL}, \mathrm{p} 0,01$, y $6,0 \pm 1,1$ a $19,4 \pm 5,9 \mathrm{ng} / \mathrm{mL}, \mathrm{p} 0,01$, respectivamente; (Figura 1). Los niveles plasmáticos basales del PIIINP no mostraron diferencias entre ambos grupos. En el grupo de mayor entrenamiento no se observó elevación signifi- cativa de este marcador $(65 \pm 11$ a $90 \pm 29 \mathrm{ng} / \mathrm{mL}, \mathrm{p}=0,10)$, mientras que, en el grupo de menor entrenamiento se observó un aumento significativo post esfuerzo $(61 \pm 16$ a $94 \pm 24 \mathrm{ng} / \mathrm{mL}, \mathrm{p}=0,01$, Figura 2).

\section{Efectos del ejercicio en biomarcadores y ventrículo} derecho. La elevación de los niveles plasmáticos de galectina-3 no se correlacionaron con la función ventricular izquierda ni derecha en ninguno de los grupos. En el grupo G2, el aumento en niveles plasmáticos del PIIINP post maratón se relacionaron significativamente con la caída en el cambio de área fraccional del ventrículo derecho (Rho: $-0,51 ; \mathrm{p}=0,04)$. Este comportamiento no se observó en los atletas más entrenados (Rho: 0,$19 ; \mathrm{p}=0,52$ ).

\section{Discusión:}

Estos resultados muestran que niveles plasmáticos de galectina-3 y PIIINP aumentan después de una carrera de maratón de $42 \mathrm{~km}$. El aumento de PIIINP fue significativamente mayor en los individuos menos entrenados y se correlacionó con una disminución en parámetros ecocardiográficos de evaluación de la función del ventrículo derecho.

Ejercicio físico y biomarcadores. Recientemente Salvagno et al, mostraron una elevación de biomarcadores de estiramiento y necrosis del miocardiocito, así como de fibrosis cardíaca luego de un esfuerzo físico intenso ${ }^{20}$. En nuestra serie se evaluaron dos biomarcadores de inflamación y fibrosis miocárdica, resultando en un significativo aumento luego de la actividad física extenuante. Estos aumentos podrían deberse al estrés celular y eventual desarrollo de fibrosis producto de una intensa actividad física. Resultados de nuestro grupo muestran que luego de un esfuerzo extenuante, los niveles plasmáticos de

\begin{tabular}{|c|c|c|c|c|c|c|}
\hline & \multicolumn{3}{|l|}{ Grupo I } & \multicolumn{3}{|l|}{ Grupo II } \\
\hline & Pre-maratón & Post-maratón & $\mathbf{P}$ & Pre-maratón & Post-maratón & $\mathbf{P}$ \\
\hline FEVI (\%) & $56 \pm 7$ & $65 \pm 6$ & 0,0001 & $54 \pm 4$ & $61 \pm 6$ & $0,0001^{*}$ \\
\hline CAF (\%) & $55 \pm 10$ & $46 \pm 22$ & 0,2 & $56 \pm 6$ & $42 \pm 18$ & $0,03^{*}$ \\
\hline TAPSE (mm) & $26 \pm 3$ & $25,5 \pm 4$ & 0,7 & $26,6 \pm 3$ & $25,5 \pm 4$ & 0,3 \\
\hline Onda S lateral & $11 \pm 2$ & $12,8 \pm 2$ & 0,07 & $11,7 \pm 3$ & $13 \pm 2$ & 0,2 \\
\hline SGL VD (\%) & $-16,7 \pm 7,4$ & $-14 \pm 10,6$ & 0,3 & $-19,3 \pm 1,5$ & $-17,8 \pm 7,4$ & 0,4 \\
\hline SLPL VD (\%) & $-26 \pm 6$ & $-23 \pm 4,6$ & 0,3 & $-24 \pm 3,6$ & $-19 \pm 4,8$ & $0,007^{\star}$ \\
\hline
\end{tabular}

FEVI: fracción de eyección del VI. CAF : cambio de área fraccional del VD. TAPSE: tricuspid annular plane systolic excursion. SGL VD: strain global longitudinal del ventrículo derecho. SLPL VD: strain longitudinal de la pared libre del ventrículo derecho. * $p<0.05$ vs pre-maraton 


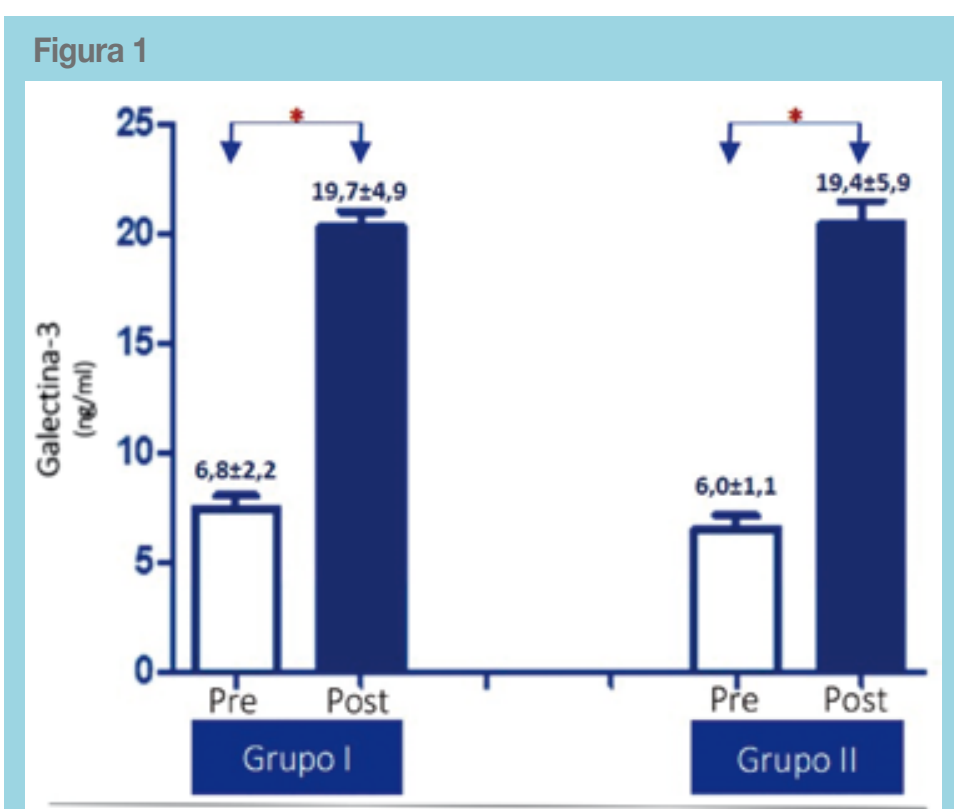

Figura 1: Niveles Plasmáticos de galectina-3 pre y post-maratón en ambos grupos de entrenamiento. " $p<0,05$ vs pre.

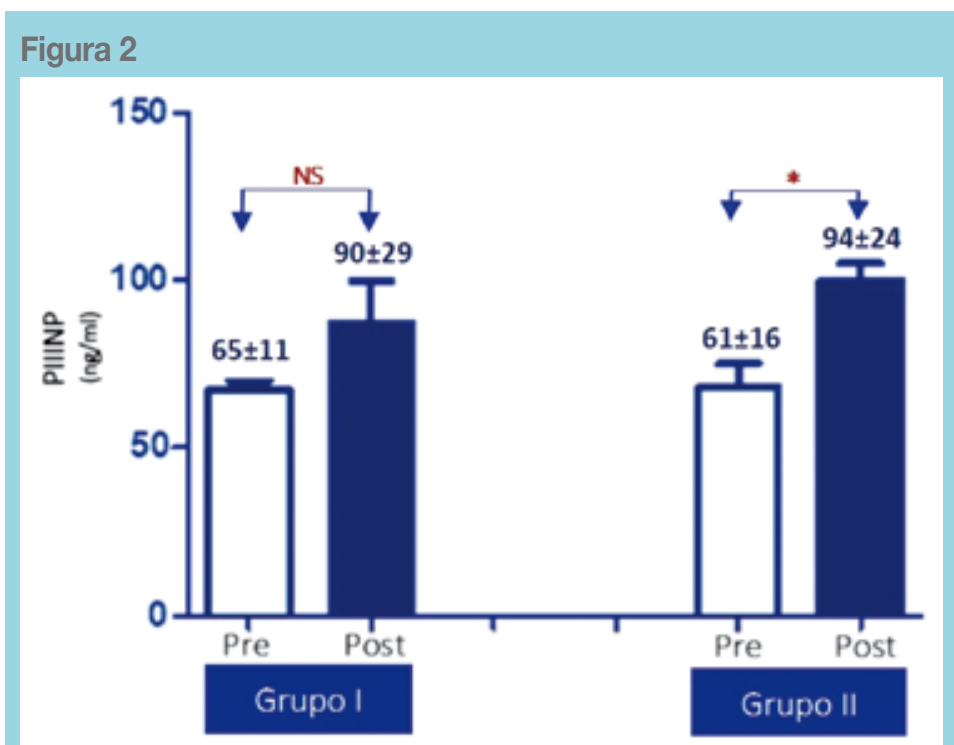

Figura 2: Niveles plasmáticos de PIIINP pre y post-maratón en ambos grupos de entrenamiento. " $p<0,05$ vs pre.

marcadores de estrés oxidativo aumentan significativamente en los atletas menos entrenados ${ }^{21}$, lo cual podría asociarse a un menor remodelado cardíaco, adaptación celular y actividad antioxidante total 22 .

Es conocido que existe una adaptación sistémica y celular al estrés inflamatorio y metabólico, generada por el ejercicio físico regular, por lo que los atletas más entrenados estarían más adaptados a cargas de ejercicio ma- yores $^{23}$. Sin embargo, las respuestas sistémicas y fisiológicas al ejercicio son complejas y no se comprenden completamente debido a que incluyen una amplia gama de cambios metabólicos, inmunológicos y hormonales ${ }^{24}$.

Biomarcadores y fibrosis. La fibrosis se caracteriza por una sobreproducción y depósito de colágeno por los fibroblastos. El PIIINP plasmático se considera un bio- 
marcador no invasivo que refleja el proceso de síntesis y degradación del colágeno, además del remodelado de la matriz extracelular cardiaca ${ }^{15,16}$. Estudios anteriores han documentado niveles elevados de PIIINP en pacientes con una variedad de anomalías que influyen en la función o la estructura cardíaca ${ }^{25}$. Hasta el momento no existen antecedentes en la literatura que relacionen los niveles séricos de PIIINP y el nivel de entrenamiento de los atletas. En este trabajo se observó un mayor aumento de PIIINP en aquellos individuos menos entrenados (menos adaptados). $\mathrm{Al}$ respecto, Hättasch et al. evidenciaron una posible correlación negativa entre el incremento de biomarcadores y el nivel de entrenamiento medido en años, observando menor aumento en los corredores más experimentados ${ }^{14}$. Probablemente con galectina- 3 no se observaron diferencias según el nivel de entrenamiento, porque ésta última está ligada a una gran cantidad de procesos biológicos como sobrevida celular, apoptosis, angiogénesis, inflamación y fibrosis y donde sus mecanismos de regulación son mucho más complejos y no solo tienen que ver con mecanismos de adaptación al esfuerzo $^{26}$.

Ejercicio intenso y ventrículo derecho. La Gerche et al mostraron que posterior a un ejercicio máximo de corta duración, el VD tiene la capacidad de aumentar la contractilidad como mecanismo compensatorio ${ }^{6}$ y que después de un ejercicio intenso de resistencia, se evidencia una disfunción aguda del mismo, evaluado tanto por ecocardiografía como resonancia magnética cardíaca, a pesar de la función aparentemente normal del ventrículo izquierdo ${ }^{27}$ Estos cambios agudos fueron reversibles en el seguimiento ${ }^{28}$. Además, en algunos atletas , con RNM se observó realce tardío a nivel ventricular derecho, lo que sugiere la presencia de fibrosis en algunos atletas ${ }^{29}$. Estos cambios estructurales y funcionales del VD han sido referido por Hein Heidbuchel, como "cardiomiopatía ventricular derecha inducida por el ejercicio", lo que se encuentra asociado a arritmias en atletas de resistencia altamente entrenados ${ }^{30}$. En nuestra serie, se observó una correlación significativa negativa entre una caída en la motilidad global del ventrículo derecho evaluada por cambio de área fraccional y la elevación de los productos de degradación del colágeno en aquellos individuos menos entrenados. Estos efectos podrían explicarse por un menor acondicionamiento lo que determina mayor estrés oxidativo e inflamación post esfuerzo en aquellos sujetos menos entrenados.

Si bien existe una concordancia significativa entre los es- tudios con respecto a la elevación de biomarcadores cardíacos inmediatamente después de un esfuerzo, su significado sigue sin estar claro. Estos son parámetros simples y no invasivos, por lo que se plantea su eventual utilidad clínica en la identificación de sujetos susceptibles y potencialmente en riesgo de un remodelado cardíaco adverso en el contexto de un entrenamiento intenso. No existe una correlación entre el ejercicio y su capacidad para mejorar la salud cardiovascular, la calidad de vida, y la longevidad en general. Se conocen claros beneficios del ejercicio aeróbico moderado en la población general en cuanto a sobrevida general, mortalidad cardiovascular y cáncer. Patil et al ${ }^{31}$ plantearon analizar la actividad física como una droga, que implica indicaciones y contraindicaciones, así como dosificación y sobredosis. Por lo que el rango de seguridad para la población general sería muy amplio y difícil de establecer. Debido a estos antecedentes y a nuestros resultados será necesario continuar evaluando estos biomarcadores de fibrosis miocárdica para ayudar al establecimiento y seguimiento del "entrenamiento" de individuos que superan la cantidad de actividad física habitualmente recomendada.

Las principales limitaciones del estudio son el pequeño tamaño muestral, considerando la dificultad técnica de un estudio con análisis inmediatamente post maratón. Los biomarcadores PIIINP y galectina 3 no son específicos para miocardio, por lo tanto, estudios experimentales que incluyan biopsias miocárdicas o estudios con resonancia magnética se requieren para visualizar la fibrosis y confirmar nuestros resultados. Otra limitación es la evaluación de la función ventricular derecha que no consideró el uso de resonancia magnética. La evaluación por ecocardiografía tiene significativa variabilidad y el strain global del ventrículo derecho incorpora la motilidad del tabique interventricular. Finalmente, el estudio no incluyó población femenina y estos hallazgos no se pueden homologar a otro tipo de disciplinas.

Como conclusión, nuestros datos muestran que el ejercicio físico de alta intensidad produce un importante aumento de biomarcadores de fibrosis miocárdica, lo cual podría tener consecuencias adversas en la estructura y función cardíaca a lo largo del tiempo. Los productos de degradación del colágeno aumentan posterior a un esfuerzo intenso e $\mathrm{n}$ sujetos con menor grado de entrenamiento se correlacionan significativamente con la caída de la función ventricular. Esto podría explicarse por una mejor adaptación asociado a menor remodelado adaptativo con respecto al grupo más entrenado 


\section{Conflictos de interés}

Los autores declaran que no tienen conflictos de intereses.

\section{Financiamiento}

Este trabajo fue financiado por los proyectos Fondecyt 1170963 (a LG) y Fondap 15130011 (a LG, MCh, MPO, SL, PFC) de CONICYT, Chile.

\section{Referencias}

1. TRIVAX JE, MCCULlOUGH PA. Reviews Phidippides Cardiomyopathy: A Review and Case Illustration. Clin Cardiol. 2012 Feb;35:69-73.

2. ZHANG Q, CHAN Y-S, LIANG Y-J, FANG F, LAM Y-Y, CHAN C-P, et al. Comparison of left ventricular reverse remodeling induced by cardiac contractility modulation and cardiac resynchronization therapy in heart failure patients with different QRS durations. Int J Cardiol. 2012;1-5.

3. FUNG JWH, YIP GWK, ZHANG Q, FANG F, CHAN JYS, LI $\mathrm{CM}$, et al. Improvement of left atrial function is associated with lower incidence of atrial fibrillation and mortality after cardiac resynchronization therapy. Heart Rhythm. 2008;5:780-6.

4. ABELA M, D'SILVA A. Left Ventricular Trabeculations in Athletes: Epiphenomenon or Phenotype of Disease? Curr Treat Options Cardiovasc Med. 2018 Oct;20:100.

5. D'ANDREA A, FORMISANO T, RIEGLER L, SCARAFILE R, AMERICA R, MARTONE F, et al. Acute and Chronic Response to Exercise in Athletes: The "Supernormal Heart". Adv Exp Med Biol. 2017;999:21-41.

6. LA GERCHE A, BURNS AT, D'HOOGE J, MACISAAC AI, HEIDBÜCHEL H, PRIOR DL. Exercise Strain Rate Imaging Demonstrates Normal Right Ventricular Contractile Reserve and Clarifies Ambiguous Resting Measures in Endurance Athletes. J Am Soc Echocardiogr. 2012 Mar;25:253-262

7. SHAVE R, OXBOROUGH D. Endurance Exercise and Myocardial Fibrosis Let Us Keep the Risk in Perspective. Circ Cardiovasc Imaging. 2016 Nov;9(11).

8. CORRADO D, ZORZI A. Sudden death in athletes. Int J Cardiol. 2017 Jun;237:67-70.
9. KONG P, CHRISTIA P, FRANGOGIANNIS NG. The pathogenesis of cardiac fibrosis. Cell Mol Life Sci. 2014 Feb;71:549-74.

10. SUGIHARA N, GENDA A, SHIMIZU M, SUEMATSU T, KITA Y, MINAMOTO M, et al. Diastolic dysfunction and its relation to myocardial fibrosis in essential hypertension. J Cardiol. 1988 Jun;18:353-61.

11. ILES L, PFLUGER H, PHROMMINTIKUL A, CHERAYATH J, AKSIT P, GUPTA SN, et al. Evaluation of diffuse myocardial fibrosis in heart failure with cardiac magnetic resonance contrast-enhanced T1 mapping. J Am Coll Cardiol. 2008 Nov;52:1574-80.

12. ZHONG X, QIAN X, CHEN G, SONG X. The role of galectin-3 in heart failure and cardiovascular disease. Clin Exp Pharmacol Physiol. 2018 Oct; 29.

13. SCIACCHITANO S, LAVRA L, MORGANTE A, ULIVIERI A, MAGI F, DE FRANCESCO GP, et al. Galectin-3: One Molecule for an Alphabet of Diseases, from A to Z. Int J Mol Sci. 2018 Jan;19.

14. HÄTTASCH R, SPETHMANN S, DE BOER RA, RUIFROK WPT, SCHATTKE S, WAGNER M, et al. Galectin-3 increase in endurance athletes. Eur J Prev Cardiol. 2014;21:1192-9.

15. HORSLEV-PETERSEN K, KIM KY, PEDERSEN LR, BENTSEN KD, ULDBJERG N, OXLUND H, et al. Serum aminoterminal type III procollagen peptide. Relation to biosynthesis of collagen type III in experimentally induced granulation tissue in rats. APMIS. 1988 Sep;96:793-804.

16. DOS SANTOS MOREIRA C, SEREJO F, ALCANTARA P, RAMALHINHO V, BRAZ NOGUEIRA J. Procollagen type III amino terminal peptide and myocardial fibrosis: A study in hypertensive patients with and without left ventricular hyper- 
trophy. Rev Port Cardiol. 2015 May;34:309-14.

17. LEE EC, FRAGALA MS, KAVOURAS SA, QUEEN RM, PRYOR JL, Casa DJ. Biomarkers in Sports and Exercise: Tracking Health, Performance, and Recovery in Athletes. J strength Cond Res. 2017 Oct;31:2920-37.

18. LANG RM, BADANO LP, MOR-AVI V, AFILALO J, ARMSTRONG A, ERNANDE L, et al. Recommendations for Cardiac Chamber Quantification by Echocardiography in Adults: An Update from the American Society of Echocardiography and the European Association of Cardiovascular Imaging. J Am Soc Echocardiogr. 2015;28:1-39.

19. RUDSKI LG, LAI WW, AFILALO J, HUA L, HANDSCHUMACHER MD, CHANDRASEKARAN K, et al. Guidelines for the echocardiographic assessment of the right heart in adults: a report from the American Society of Echocardiography endorsed by the European Association of Echocardiography, a registered branch of the European Society of Cardiology, and the Canadian Society of Echocardiography. J Am Soc Echocardiogr. 2010 Jul;23:685-8.

20. SALVAGNO GL, SCHENA F, GELATI M, DANESE E, CERVELLIN G, GUIDI GC, et al. The concentration of high-sensitivity troponin I, galectin-3 and NT-proBNP substantially increase after a 60-km ultramarathon. Clin Chem Lab Med. 2014 Feb;52:267-72.

21. VEGA J, CONTRERAS-BRICEÑO F, SAAVEDRA R, FERNÁNDEZ R, HERRERA S, SALINAS M, et al. Entrenamiento físico de alta intensidad en maratonistas produce mayor remodelado cardíaco y reduce respuesta de estrés oxidativo High-intensity training in marathon runners increases cardiac remodeling and diminishes oxidative stress response. Rev Chil Cardiol 2018; 37: 93-103.

22. MUNOZ D, BARRIENTOS G, ALVES J, GRIJOTA FJ, ROBLES MC, MAYNAR M. Oxidative stress, lipid peroxidation indexes and antioxidant vitamins in long and middle distance athletes during a sport season. J Sports Med Phys Fitness. 2018 Dec;58:1713-1719
23. FISCHER CP. Interleukin-6 in acute exercise and training: what is the biological relevance? Exerc Immunol Rev. 2006;12:6-33.

24. SCHILD M, EICHNER G, BEITER T, ZÜGEL M, KRUMHOLZ-WAGNER I, HUDEMANN J, et al. Effects of Acute Endurance Exercise on Plasma Protein Profiles of Endurance-Trained and Untrained Individuals over Time. 2016;2016.

25. WANG TJ, LARSON MG, BENJAMIN EJ, SIWIK DA, SAFA $\mathrm{R}$, GUO C, et al. Clinical and echocardiographic correlates of plasma procollagen type III amino-terminal peptide levels in the community.

26. DONG R, ZHANG M, HU Q, ZHENG S, SOH A, ZHENG Y et al. Galectin-3 as a novel biomarker for disease diagnosis and a target for therapy. Int J Mol Med. 2018;41:599-614.

27. ELLIOTT AD, LA GERCHE A. The right ventricle following prolonged endurance exercise: are we overlooking the more important side of the heart? A meta-analysis. Br J Sports Med. 2015 Jun;49:724-9.

28. HEIDBUCHEL H, PRIOR DL, LA GERCHE A. Ventricular arrhythmias associated with long-term endurance sports: what is the evidence? Br J Sports Med . 2012 Nov 1;46

29. LA GERCHE A, CLAESSEN G, DYMARKOWSKI S, VOIGT JU, DE BUCK F, VANHEES L, et al. Exercise-induced right ventricular dysfunction is associated with ventricular arrhythmias in endurance athletes. Eur Heart J. 2015;36:19982010 .

30. HEIDBUCHEL H, HOOGSTEEN J, FAGARD R, VANHEES L, ECTOR H, WILLEMS R, et al. High prevalence of right ventricular involvement in endurance athletes with ventricular arrhythmias. Role of an electrophysiologic study in risk stratification. Eur Heart J. 2003 Aug;24:1473-80.

31. PATIL HR, O'KEEFE JH, LAVIE CJ, MAGALSKI A, VOGEL RA, MCCULLOUGH PA. Cardiovascular damage resulting from chronic excessive endurance exercise. Mo Med. 2012;109:312-21. 